\title{
High Efficiency Ka-Band Spatial Combiner
}

\author{
Davide Passi ${ }^{1}$, Alberto Leggieri ${ }^{1}$, Franco Di Paolo ${ }^{1}$, \\ Marco Bartocci ${ }^{2}$, Antonio Tafuto ${ }^{2}$, and Antonio Manna ${ }^{2}$ \\ ${ }^{1}$ Department of Electronic Engineering, University of Rome Tor Vergata, Rome, Italy \\ ${ }^{2}$ Elettronica SpA, Via Tiburtina Valeria Km. 13,7 - 00100 Rome, Italy \\ *corresponding author, E-mail: davide.passi@uniroma2.it
}

\begin{abstract}
A Ka-Band, High Efficiency, Small Size Spatial Combiner (SPC) is proposed in this paper, which uses an innovatively matched quadruple Fin Lines to microstrip (FLuS) transitions. At the date of this paper and at the Author's best knowledge no such FLuS innovative transitions have been reported in literature before. These transitions are inserted into a WR28 waveguide T-junction, in order to allow the integration of 16 Monolithic Microwave Integrated Circuit (MMIC) Solid State Power Amplifiers (SSPA's). A computational electromagnetic model using the finite elements method has been implemented. A mean insertion loss of $2 \mathrm{~dB}$ is achieved with a return loss better the $10 \mathrm{~dB}$ in the $31-37 \mathrm{GHz}$ bandwidth.
\end{abstract}

\section{Introduction}

The Spatial Power Combiner (SPC) Technology [1-7], even if in its theory it is quite consolidated, in these last years it is knowing an increasing interest. In fact, especially in the field of wideband airborne and spacecraft telecommunications where size and reliability is a major concern, there is an increasing need of an SSPA alternative to high power Vacuum Tubes (VT's) microwave amplifiers, that are typically based on travelling wave tube (TWT) amplifiers. In fact, even if a single VT has higher power output than a single transistor, the reliability and low voltage operation [8-9] of the Solid State technology give a strong tendency to use this last respect the VT technology. However, the main problem for SSPA to compete with Vacuum Tube Power Amplifier (VTPA) is to combine many single active devices to try to reach a signal power level at least comparable with that produced by VTPA.

Considering only the output signal power, SS vs. VT competition makes sense only in some frequency bands and for some power levels. For example, in the range of millimeter waves (mmW's) or sub-mmW's and signal power levels greater than kilowatt $(\mathrm{KW})$ no SS vs. VT competition exists: here, VT's like Gyrotrons or Free
Electron Lasers (FEL's) have the dominance [10-13]. Different is the case when the competition is in the microwave range and signal power levels under some KW: here, SSPA's based on the SPC technology can compete with VTPA's, at least in some applications. Even if the signal power level of a VTPA can only be barely reached with SSPA combining many transistors, another big advantage of this last is its graceful degradation: a failure in a VTPA is a failure of the whole system, while a failure of a single transistor inside a combined SSPA is highly mitigated, at least when many transistors are combined to reach the desired signal power level.

The traditional approach to obtain a significant power output using SSPA's is the binary combining of many transistors through microstrip Transmission Lines [14-15]. This solution is limited to the losses which each printed transmission line (TL) combiner inserts. Binary combining, in addition, limits the number of devices to a power of two, and the designer may be forced to combine more than the necessary devices.

A complete different approach is the Spatial Power Combiner Amplifier. In this case, power dividing and combining is performed in a parallel way, rather than in a serial way, as shown in figure 1 , and losses are little dependent with the number of used PA's [16].

A fundamental block of the SPA is the Spatial Power Combiner (SPC), which is a passive reciprocal device that for this reason can also be called Spatial Power Divider (SPD): the SPC (or SPD) performs a parallel combining (or dividing) operation of many RF signals, thus reducing the combining (or dividing) loss to a minimum value. To realize an SPA, the RF signal entering a spatial region is captured by opportune EM probes which divide the signal in a parallel way: this function realizes the SPD. Then, these signals feed SSPA's, and the amplified signals are summed in space through similar EM probes and, again, in a parallel way: this function realizes the SPC. Even if the functions that SPC and SPD realize are different and even if in any SPA both of them are needed, quite often the SPA is anyhow named SPC. Since the SPA loss is rather constant with the number of amplifiers, due the parallel dividing and combining functions, amplifier size limits the combiner area. In addition, other size of the SPA is 
dedicated to the bias network and heat sink systems, which are especially important in order to avoid performance degradation and even device failure.

In this work, the design and simulation a novel high performance and small size SPC working in Ka Band is presented, interfacing with standard WR2 8 waveguides. It employs an innovative matched quadruple antipodal Fin Line transition: at the Author's best knowledge, no such transition as ever appeared in literature before. Such innovative SPC allows the in-phase combination of 16 MMIC PA's with low losses, then achieving a high combining efficiency. Several circuital and technological solutions have been adopted. In order to reduce the combining loss and size and to reach wideband matching, exponential FLuS transitions have been considered. In order to improve the operative band, a parasitic void has been implemented by inserting an anti-resonance metal in the antipodal FLuS transition profile [17]. The simulation of the structure gives an insertion loss of less than $3 \mathrm{~dB}$ when combining $16 \mathrm{MMIC}$ 's, in $32-38 \mathrm{GHz}$ band, with a maximum gain variation of $\pm 0.5 \mathrm{~dB}$ and return loss better than $10 \mathrm{~dB}$ in the whole bandwidth.

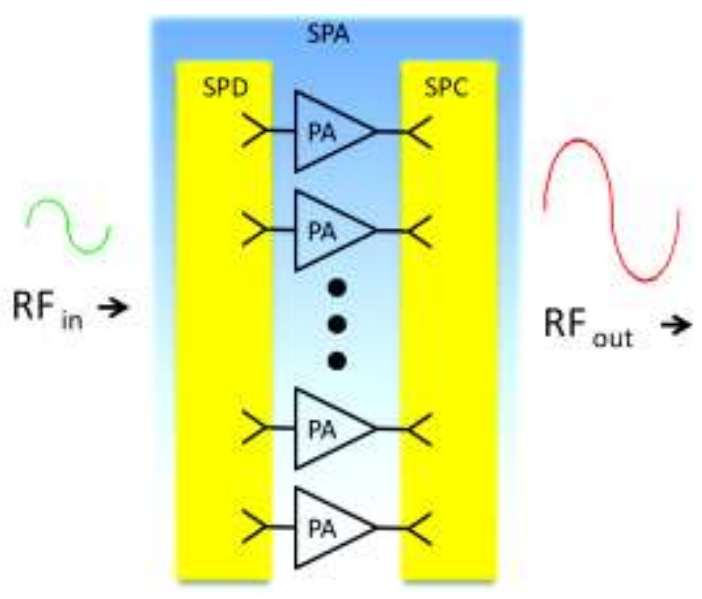

Figure 1: The general concept of Spatial Power Amplifier.

\section{The quad fin-line to microstrip transition}

The SPC unit considered in this paper uses a standard WR28 waveguide flange at input and output. In order to transform the electromagnetic field inside such waveguide, i.e. the fundamental $\mathrm{TE}_{10}$ mode, so that the field can be intercepted by the MMIC's, we use four $\mathrm{Al}_{2} \mathrm{O}_{3}$ cards, each one containing quadruple (quad) FLuS transitions.

The Fin Line taper in each FLuS can be used as a broad-band impedance transformer between the waveguide and MMIC amplifier, since the characteristic impedance at the waveguide is different to the impedance required by the PA input port matching condition. Therefore a portion of incident energy, being initially in $\mathrm{TE}_{10}$ waveguide mode inside the WR28, is efficiently converted into a quasi-TEM (qTEM) microstrip mode by the FLuS.

\subsection{Fin taper profile}

In order to reduce the combining loss, the shape of the Fin Line taper has to be chosen properly so that return loss can be minimized within the frequency band of interest. Several spatial profiles of FL can be implemented: exponential, parabolic, sine, sine squared, cosine and cosine squared taper [11]. The former is chosen to ensure the smoothness of the taper, whereby very good matchedload is readily available for the Waveguide, because this transition indeed reflects a small amount of incident power. The spatial profile of an exponential Fin taper is given by (1):

$$
w(z)=w_{0} \exp \left\lfloor\frac{z}{l} \ln \left(w_{f}\right)\right\rfloor,
$$

where $w_{0}$ stands for initial taper width, $w_{f}$ for the final one and $l$ is the total length of the transition [18]. The position dependent impedance of an exponential Fin taper is given by (2):

$$
Z(z)=Z_{0} \exp \left\lfloor\frac{z}{l} \ln \frac{Z_{1}}{Z_{2}}\right\rfloor,
$$

where $Z_{2}$ is the impedance to match, $Z_{1}$ is the matching impedance, generally $50 \Omega$, and $z$ is the coordinate of the longitudinal axis. By applying the small reflections theory, the spatial profile $w(z)$ can be taken as the uniform TL characteristic impedance $Z(z)$. From Riccati's equation it is possible to find the reflection coefficient trend [19] with respect the product $\beta l$, given by (3).

$$
\Gamma=\frac{1}{2} e^{-j \beta l} \ln \left\lfloor\frac{Z_{1}}{Z_{2}}\right\rfloor \frac{\sin (\beta l)}{\beta l},
$$

Microstrip Lines were designed following Hammerstad formulas reported in [20].

\subsection{Antipodal configuration}

In order to obtain great system integration, antipodal configuration of FLuS transition is an attractive solution, since transitions are in line with the direction of propagation and can be easily manufactured on inexpensive soft substrates or hard substrates, using standard printed circuit board techniques or sputtering techniques. This configuration expects two metal FL transition sheets placed among a dielectric substrate to lead hot and ground planes. The profile of one sheet is the dual mirrored of the other.

In this work, four antipodal Fin Lines are realized on $\mathrm{Al}_{2} \mathrm{O}_{3}$ substrate, and at the Author's best knowledge it is the first time that such antipodal quadruple structure is realized. Other quadruple Fin-Line structures have been reported [21], but these are not in antipodal configuration. 
The proposed structure is reported in figure 2, in which top metal sheets are in green, bottom ones are in yellow.

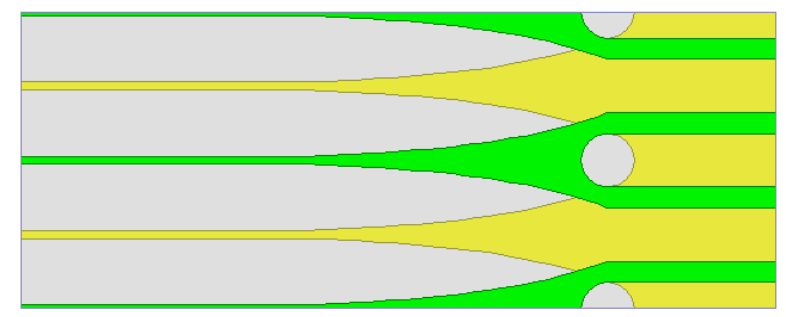

Figure 2: Quadruple Fin Line in Antipodal Configuration.

\subsection{Band widening}

The problem, when using the antipodal configuration, is the difficult to obtain broadband response, since the transition creates a set of resonant modes which limit its useful bandwidth. Near the terminal microstrip section of Antipodal FLuS transition, where quasi TEM mode are being generated, $\mathrm{TE}_{10}$ mode tends to cutoff, and evanescent modes are being created, storing inductive energy. The FinLine discontinuity appears to the evanescent modes as a parasitic slot in a bisecting metal sheet. In this region electric field will be created, storing capacitive energy. When the capacitive energy created by the slot equals the inductive energy stored in the evanescent modes, a resonance will occur. In order to avoid this resonance, the Fin-Line discontinuities can be designed so as to break the slot electric field lines [17]. An useful solution has been applied; it consists to model a circular section through the metal sheet in the area where the parasitic slot might create. The quadruple Fin Line with resonance absorption is shown in figure 3.

Obviously the taper efficiency varies with its profile. The microstrip tapers have meandering shapes so that the taper lengths and the resulting phase differences remain the same among them.

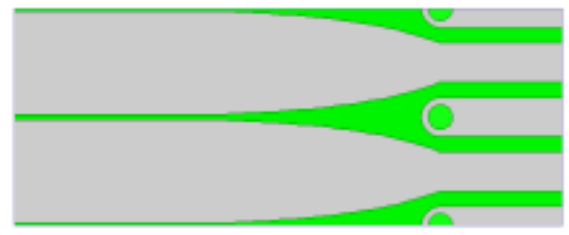

Figure 3: Quadruple Fin Line with resonance absorption.

To improve the return loss at the waveguide when loaded by the Fin Lines, usually dielectric Quarter Wave Transformers (QWT's) may be provided [18]. In the actual case of four antipodal Fin Lines, an innovative structure of QWT has been adopted, which we name "parallel QWT" (P-QWT); also in this case, at Author's best knowledge no such innovative structure has ever been previously reported in literature. The P-QWT structure is reported in figure 4.

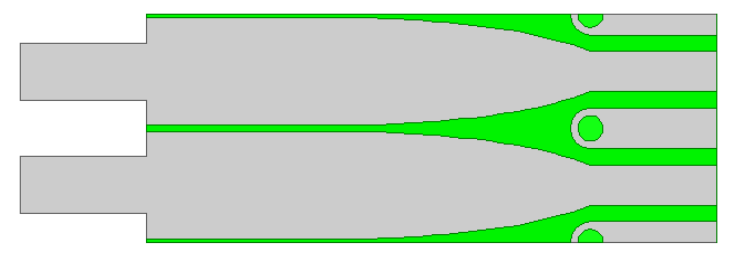

Figure 4: The complete, innovative, quadruple FLuS.

The P-QWT has a very important role, i.e. that to make symmetric the currents on the substrate, so that the quadruple Fin Lines receive the same quantity of electromagnetic fields. In only a QWT were used, there would be a great amount unsymmetrical dividing between the four microstrips ports. A figure of the currents in the substrate with the P-QWT is shown in the following figure 5. In figure 6 we show the splitting at the four microstrips ports. In the next section we will describe the design of the whole Spatial Combiner.

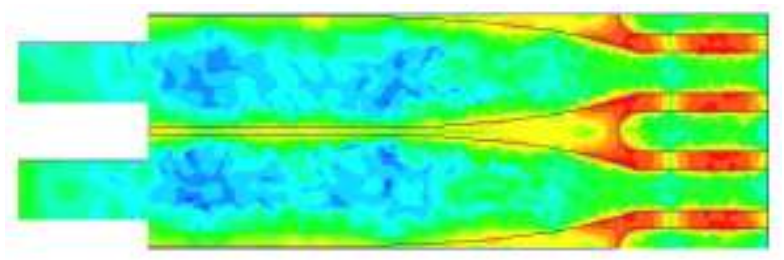

Figure 5: The symmetry of currents inside the quadruple FLuS, due the use of P-QWT.

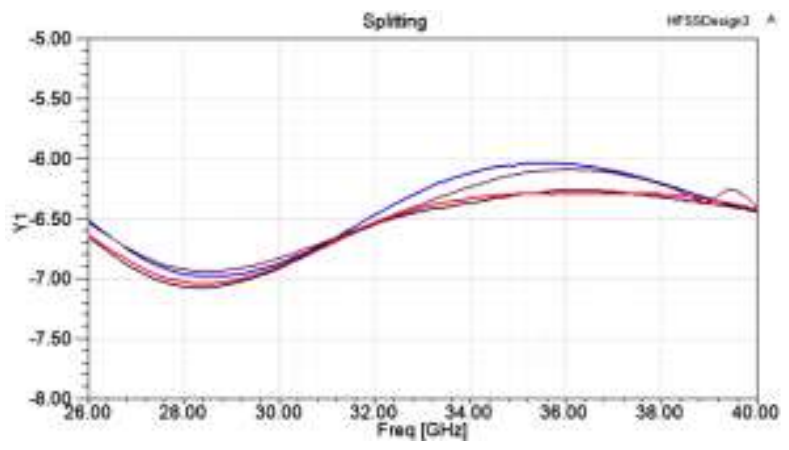

Figure 6: The perfect splitting on the quadruple innovative FLuS.

\section{The proposed Ka-band spatial combiner}

In this work, a novel High Performance and small size SPC working in $\mathrm{Ka}$ Band is presented, which employs the innovative matched quadruple Fin Line transition discussed before. This SPA has been designed to use standard WR28 flanges and to employ 16 MMIC HPA's operating at these WG frequencies, exactly the type TGA2575 produced by Triquint. At the amplifier input, a Waveguide T-junctions in E-plane is used to split the signals in two WR28 arms. The 
branches of T-Junction have designed with four-step Chebyshev transformer in order to obtain small reflections to the input port in $\mathrm{Ka}$ band.

First, the iris has been optimized to obtain the minimum return loss around $35 \mathrm{GHz}$, by setting the width $\mathrm{W}_{0}$ of the Tjunction output branches to $1.55 \mathrm{~mm}$. Then, adapting the procedure reported in [19] to rectangular WG case, we obtain the following figures of merit:

$A=\frac{1}{2} \rho_{m}^{-1} \ln \frac{Z_{L}}{Z_{0}}=\frac{1}{2} \rho_{m}^{-1} \ln \frac{W_{5}}{W_{0}}=41.519$,

$\Delta f=f_{0} \frac{4}{\pi}\left(\frac{\pi}{2}-\vartheta_{m}\right) \cong 28 G H z$,

$\vartheta_{m}=0.934$

where $A$ is the attenuation, $\rho_{m}$ is the maximum passband reflection coefficient, $\mathrm{W}_{5}$ is the width of WR28 $(3.556 \mathrm{~mm})$, $\vartheta_{m}$ is the electric length since we have the equal-ripple characteristic; finally $\Delta f$ is the passband obtained.

The width of each section is obtained from (7) and reported in table 1 .

$$
W_{i}=W_{0} \exp \left(\sum_{k=0}^{i-1} 2 \rho_{k}\right)
$$

where $\rho_{k}$ are the calculated Chebyshev coefficients reported in table 2 .

Table1: Width of Chebyshev transformer sections(mm).

\begin{tabular}{|c|c|c|c|c|c|}
\hline $\mathbf{W}_{\mathbf{0}}$ & $\mathbf{W}_{\mathbf{1}}$ & $\mathbf{W}_{\mathbf{2}}$ & $\mathbf{W}_{\mathbf{3}}$ & $\mathbf{W}_{\mathbf{4}}$ & $\mathbf{W}_{\mathbf{5}}$ \\
\hline 1.550 & 1.679 & 2.067 & 2.665 & 3.279 & 3.556 \\
\hline
\end{tabular}

Table 2: Calculated coefficients of Chebyshev transformer.

\begin{tabular}{|c|c|c|c|c|}
\hline $\boldsymbol{\rho}_{\mathbf{0}}$ & $\boldsymbol{\rho}_{\mathbf{1}}$ & $\boldsymbol{\rho}_{\mathbf{2}}$ & $\boldsymbol{\rho}_{\mathbf{3}}$ & $\boldsymbol{\rho}_{\mathbf{4}}$ \\
\hline 0.0401 & 0.1038 & 0.127 & 0.1038 & 0.0401 \\
\hline
\end{tabular}

For each arm, the fundamental mode $\mathrm{TE}_{10}$ entering inside such WG couples to two $\mathrm{Al}_{2} \mathrm{O}_{3}$ substrate cards, each one carrying four antipodal FLuS's, described above, so that each WG arm holds a total of 8 MMIC's. In practice, each FLuS transforms the $\mathrm{TE}_{10} \mathrm{WG}$ field in a q-TEM $\mu \mathrm{S}$ mode, ready to be captured and amplified by MMIC's. Such transformation is accompanied by field rotation. Considering the electric field $\mathrm{E}$ and the reference system in figure 2 , this rotation consists to rotate the WG $\mathrm{E}$ field aligned along $\mathrm{y}$ to the $\mathrm{q}$-TEM $\mathrm{E}$ field aligned along $\mathrm{z}$. This rotation is reported in the following figure 7.

Microstrip lines are used to connect the MMIC's to the quadrifins, since the dimensions of these MMIC's are much wider than what available for the WR28 waveguide. At the output, a similar microstrip lines and T-junction is used to combine the signal amplified by the MMIC's.

To allow heat dissipation without mismatch between coefficients of thermal expansion (CTE) for GaAs and conductors, MMIC's are brazed onto CuMo heat spreader and the whole assembly is brazed onto copper carriers. In figure 8 we report the complete CAD drawing of the KaBand SPC amplifier, including 16 SSPA MMIC's: top cover is removed for a better view. From such image we recognize the bulk of the SPC made of Aluminum, the innovative quadruple fin lines with the innovative P-QWT, the copper carriers, the equalization lines and the MMIC's with their DC feed capacitors placed on CuMo heat spreader. The size of this SPC is $90 \times 90 \times 27 \mathrm{~mm}^{3}$, but size can be still reduced.

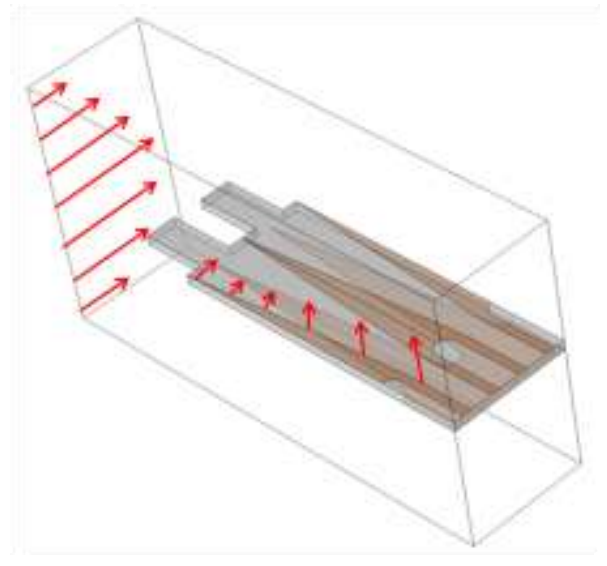

Figure 7: Electric field rotation performed by each FLuS.

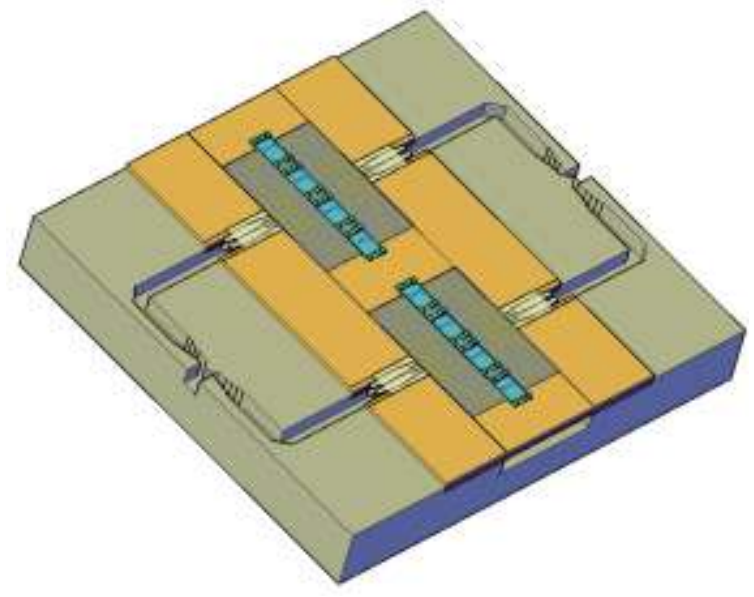

Figure 8: The complete Ka-Band SPC Amplifier: top cover removed for simplicity of view.

\section{Computational electromagnetic model}

After the analytical design of this SPA, as described above, a computational electromagnetic model using the finite elements method has been implemented on HFSS version 15 of Ansys. The SPC has been optimized and finally simulated. Such innovative SPC allows the inphase combination of 16 MMIC PA's, and several simulations problems need to be solved, such as a large amount of tetrahedrons in meshing steps and several resonance occurrences. The selection of the simulated geometry must always to be carefully determined, to avoid 
undesired lack of software convergence or bad results [22].

At last about 230 thousands tetrahedrons versus a million ones have been obtained, resulting in a reliable modeling which has ensured correct results. This improvement was achieved by defining two symmetry planes, which are a Perfect Magnetic Conductor (PMC) and a Perfect Electric Conductor (PEC). Furthermore, since a good discretization of the Fin taper profile was of fundamental importance, the exponential profile was approximated with a discrete number of points such that the different segment obtained were smaller than $\lambda / 20$ at the highest frequency of the operative band. In this way the discretization doesn't affect the performance but reduces the computational complexity.

By adopting these strategies and by enabling the Iterative Solver, 2.2 GB of memory have been employed, instead of $10 \mathrm{~GB}$ ones necessary without this reduction. The 3D simulated structure is shown in figure 9, from which we recognize that one quarter of the structure shown in figure 8 can be analyzed with the software.

The simulated S-parameters are given in figures 10 , $\mathrm{s}_{11}$, and $11, \mathrm{~s}_{21}$. By observing such images we note that the proposed WR28 SPC ensures a maximum insertion loss of $2.2 \mathrm{~dB}$ and a minimum Return Loss of $10 \mathrm{~dB}$ in the whole 31-37 GHz band.

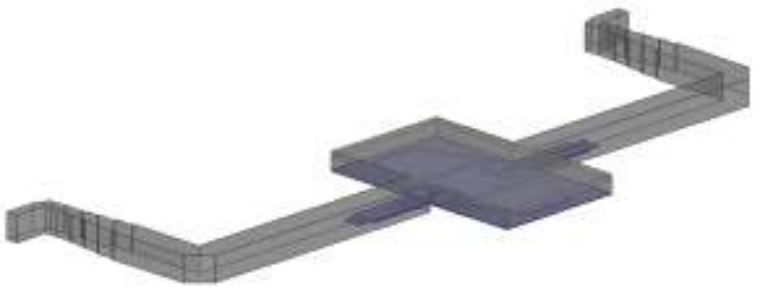

Figure 9: Simulated 3D quarter section of the WR28 SPC.

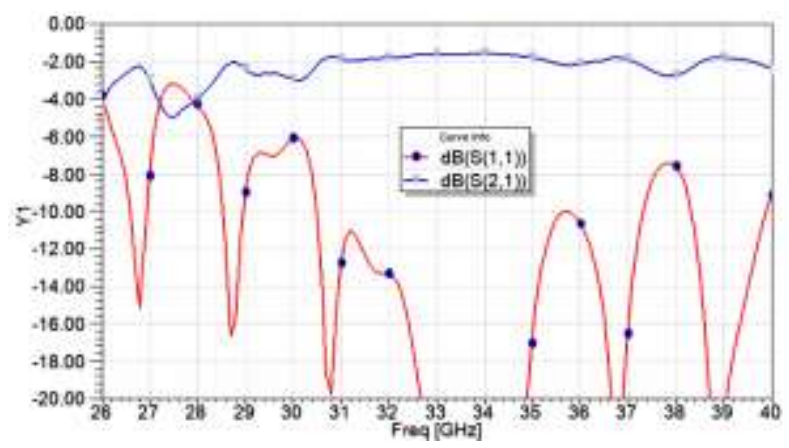

Figure 10: Simulated full WR28 band SPC reflection coefficient and losses in $\mathrm{dB}$.

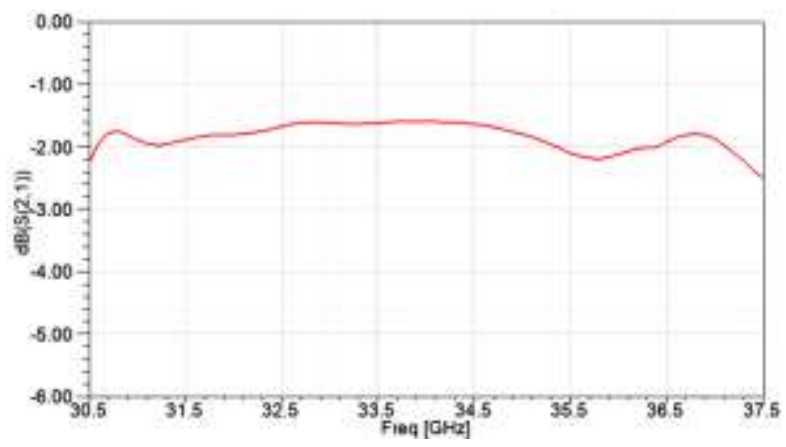

Figure 11: Simulated SPC Insertion Loss in $d B$, in the operating bandwidth.

\section{Conclusions}

The proposed SPC represents an innovative solution, in order to increase available power outputs, small size and low cost for a Ka Band Power Amplifier. An innovative structure, never found before of this work, of quadruple fin line which employs and innovative parallel quarter wave transformer has been studied and simulated, which allows the combination of 16 SSPA MMIC's. A reliable computational electromagnetic model based on the finite elements method has been implemented and the results have shown an operating bandwidth from $31 \mathrm{GHz}$ to 37 $\mathrm{GHz}$, with a mean insertion loss of $2 \mathrm{~dB}$ and minimum return loss of $10 \mathrm{~dB}$ in the whole bandwidth.

\section{References}

[1] A. Leggieri, G. Orengo, D. Passi, F. Di Paolo, The Squarax Spatial Power Combiner, Progress In Electromagnetic Research C, Vol. 45, p 43-55, 2013.

[2] I. Russo, L. Boccia, G. Amendola, G. Di Massa, Parametric Analysis of Grid Amplifiers, Proceedings of European Microwave Week 2008, Amsterdam, p: 1326-1329.

[3] D. An, X. Li, J. Mou, X. Liv, A new type of Ka-Band Waveguide-Based Power Combining Structures, Proceedings of ICMMT 2008, 21-24 April 2008, Nanjing, China, p: 1-4.

[4] I. Russo, L. Boccia, G. Amendola, H. Shumacher, Compact Hybrid Coaxial Achitecture for $3-10 \mathrm{GHz}$ UWB Quasi-Optical Power Combiner, PIER, Progress In Electromagnetic Research, Vol. 122, 2012, p:77-92.

[5] D. Lopz, L. Bortoli, J-P Fraysse, D. Langrez, J.F. Villemazet, J.L Cazaux, G. Soubercaze-Pun, L. Lapierre, HPA Module for Active Antenna Applications, Proceedings of 5th European Microwave Integrated Circuit Conference, Paris, 2728 Sept. 2010, p: 404-407

[6] M. P. DeLisio and R. A. York, Quasi-optical and spatial power combining, IEEE Trans. on MTT, Vol. 50, No. 3, Mar. 2002. 
[7] Nai-Shuo Cheng, Pengcheng Jia, David B. Rensch, and Robert A. York, A 120-W X-Band Spatially Combined Solid-State Amplifier, IEEE Trans. on MTT, Vol. 47, No. 12, December 1999.

[8] R. S. Symons, Tubes: Still vital after all these years, IEEE Spectrum, Apr. 1998, p: 52-63.

[9] E. Barbour, The Cool Sound of Tubes, IEEE Spectrum, Aug. 1998, p: 24-35.

[10]B. Levush, D.K. Abe, J.P. Calame, B.G. Danly, K.T. Nguyen, E.J. Dutkowski, R.H. Abrams, R.K. Parker, Vacuum Electronics: Status and Trends, 2007 IEEE Radar Conference, p:971-976

[11] C.M. Armstrong, The vitality of vacuum electronics, IEEE 14th International Vacuum Electronics Conference (IVEC), 2013, p:1-3

[12] J.H.Booske, R.J.Dobbs, C.D.Joye, C.L.Kory, G.R.Neil, P.Gun-Sik, P.Jaehun, R.J.Temkin, Vacuum Electronic High Power Terahertz Sources, IEEE Transactions on Terahertz Science and Technology, Vol:1, Issue: 1, 2011, p:54-75

[13] J.X.Qiu, B.Levush, J.Pasour, A. Katz, C.M. Armstrong, D.R. Whaley, J. Tucek, K. Kreischer, D. Gallagher, Vacuum tube amplifiers, IEEE Microwave Magazine, Vol:10, Issue:7, 2009, p:38-51

[14] K.J.Russel, Microwave Power Combining Techniques, IEEE Trans. on MTT, Vol. MTT-27, N. 5, May 1979, p.472-478.

[15]K. Chang, C. Sun, Millimeter-Wave Power Combining Techniques, IEEE Trans. on MTT Vol. MTT-31, N. 2, Feb 1983, p.91-107.

[16] M. P. DeLisio and R. A. York, Quasi-optical and spatial power combining, IEEE Trans. on MTT, Vol. 50, No. 3, Mar. 2002.

[17] George E. Ponchak, Alan N. Downey, A New Model for Broadband Waveguide to Microstrip Transition Design, NASA Technical Memorandum 88905, Lewis Research Center Cleveland, Ohio, December 1986.

[18]B. Bhat, S.K. Koul, Analysis, Design and Applications of Fin Lines, Artech House, 1987.

[19] R.E. Collin, Foundations for Microwave Engineering, McGraw-Hill International Editions, 1992, ISBN 007-011811-6.

[20]F. Di Paolo, Networks and Devices Using Planar Transmission Lines, CRC Press LLC, 2000, ISBN 08493-1835-1.

[21] Nai-Shuo Cheng, Pengcheng Jia, David B. Rensch, and Robert A. York, A 120-W X-Band Spatially Combined Solid-State Amplifier, IEEE Trans. on MTT, Vol. 47, No. 12, December 1999.

[22] D. Passi, A. Leggieri, F. Di Paolo, Computational Electromagnetic design of the Squarax Spatial Power Combiner, Ansys UGM 2014, Milano, Italy. 